\title{
Factores de riesgo asociados a traumatismo al nacimiento
}

\author{
José Mauro Madi ${ }^{1}$, Rodrigo Vieira Jacobi ${ }^{1}$, Breno Fauth de Araújo ${ }^{1}$, Camila Viecceli \\ Viecceli $^{a}$, Daniel Ongaratto Barazzetti ${ }^{a}$, Gabriela Pavan Pavan. ${ }^{a}$ \\ ${ }^{1}$ División de Ginecología y Obstetricia, Hospital General de Caxias do Sul, Facultad de Medicina, Universidad de Caxias \\ do Sul, Rio Grande do Sul, Brasil.
}

a Alumno, Facultad de Medicina, Universidad de Caxias do Sul, Rio Grande do Sul, Brasil.

\section{RESUMEN}

Antecedentes: Se define trauma al nacimiento las lesiones sufridas por el feto durante el trabajo de parto o expulsión. Objetivo: Identificar los factores de riesgo asociados a lesiones originadas durante el nacimiento en recién nacidos. Método: Estudio casos y controles, realizado en el período de julio/2004 a diciembre/2005, en la División de Ginecología y Obstetricia del Hospital General de Caxias do Sul/ Facultad de Medicina, Universidad de Caxias do Sul, RS, Brasil. Fueron analizadas variables maternas, del parto y fetales, utilizando el programa estadístico SPSS versión 19.0. Los factores que obtuvieron nivel significativo $<0,10$ en los análisis bivariado fueron insertas en la regresión logística. Se utilizó el modelo de entrada por bloques (block entry) para selección del modelo final de la regresión. Resultados: En el período citado nacieron 2.137 infantes, 26 de ellos $(1,2 \%)$ sufrieron trauma al nacimiento. La fractura de clavícula fue la lesión más frecuente $(n=14 ; 53,8 \%)$, seguida del cefalohematoma $(n=5 ; 19,2 \%)$. Las variables gasométricas no presentaron diferencia estadística. En la regresión logística, las únicas variables independientes asociadas a traumatismo al nacimiento fueron parto vaginal (OR-A: 11,08 ; IC95\%: 2,45-49,98; $p=0,002$ ) y perímetro torácico $\geq 33 \mathrm{~cm}$ (OR-A: 3,36; IC95\%: 1,35-9,73; $p=0,010)$. Conclusión: Los factores de riesgo asociados a lesiones durante el nacimiento involucran el parto vaginal y el perímetro torácico igual o superior a $33 \mathrm{~cm}$.

\section{PALABRAS CLAVE: Traumatismo, factores de riesgo, estudios de casos y controles, embarazo}

\section{SUMMARY}

Background: Obstetrics injuries can be defined as fetal lesions suffered by the fetus during labor or expulsion. Objective: Identify risk factors for fetal birth trauma. Method: Case-control study conducted from July 2004 to December 2005. We analyzed maternal and fetal variables using software SPSS 19.0. The variables showing a significance level $<0.10$ in the bivariate analysis were included in the logistic regression analysis. We used the block entry model for selection of the final regression model. Results: During these period, we identified 2137 births and $26(1.2 \%)$ were related to fetal birth injury. Clavicle fracture was the most frequent injury $(n=14 ; 53.8 \%)$, followed by cephalohematoma $(n=5 ; 19.2 \%)$. Umbilical cord blood gas analysis did not show statistical significance. In the regression analysis of the variables that had significance level $<0.10$, the only independent variables associated with fetal birth injury were vaginal delivery (OR-A: $11.08 ; 95 \% \mathrm{Cl}$ : 2.45-49.98; $p=0.002$ ) and thorax circumference $\geq 33 \mathrm{~cm}$ (OR-A: 3.36; 95\%Cl: 1.35-9.73; $p=0.010$ ). Conclusion: The risk factors for fetal birth injury were vaginal delivery and chest circumference equal to or larger than $33 \mathrm{~cm}$. Other variables were not associated with the outcome in discussion.

KEY WORDS: Fetal injury, risk factors, case-control study, pregnancy 


\section{INTRODUCCIÓN}

Se define trauma al nacimiento las injurias fetales sufridas por los tejidos del recién nacido que se produce durante el trabajo de parto o expulsión $(1,2)$. La incidencia del evento se sitúa alrededor de $1 \%$ de todos los nacimientos ocurridos en maternidades asistidos por médico obstetra. La mortalidad neonatal dependiente del trauma al nacimiento, se sitúa en torno al $8 \%$ de los óbitos entre los recién nacidos ( $\mathrm{RN})$ a término (3).

Entre los factores que se han asociado a la presentación de lesiones durante el nacimiento se encuentran la macrosomia fetal, principalmente en fetos con peso superior a 4.500 gramos $(4,5)$, trabajo de parto prolongado, presentación pélvica, desproporción cefalopélvica y aplicación de fórceps (6-10). También se asocian a procedimientos dianósticos y terapéuticos, y el empleo intempestivo de substancias generadoras de taquisistolia e hipertonía uterina (3).

Los traumas más frecuentes son la fractura de clavícula, las parestesias braquiales y facial (10-12), el cefalohematoma y los traumas/heridas en la cabeza, rostro y brazo del RN $(13,14)$. El objetivo del presente estudio fue identificar la incidencia de trauma al nacimiento y sus factores de riesgo asociados.

\section{PACIENTES Y MÉTODOS}

Estudio caso-control realizado en el período de julio/2004 a diciembre/2005, en los Servicios de Ginecología/Obstetricia y de Neonatología del Hospital General, Fundación Universidad de Caxias do Sul, hospital universitario público, de nivel terciario de atención, que atiende solamente pacientes del Sistema Único de Salud. Los servicios citados son referencia para gestantes de alto riesgo de la región Nordeste del Estado, que engloba 50 municipios. Esto resulta en la atención de un gran número de $\mathrm{RN}$ y gestantes de alto riesgo oriundo de otras ciudades, que contribuye actualmente con más del $40 \%$ de las camas obstétricas y neonatales.

Los casos de traumatismo al nacimiento fueron identificados por los médicos que asistieron al parto o los de la Unidad de Internación Obstétrica, por ocasión de la visita protocolar puerperal, y por los neonatólogos de la Unidad de Tratamiento Intensivo Neonatal, cuando había internación de los RN en esa unidad. Todas las informaciones obtenidas formaban parte de un protocolo previamente estructurado.

Fueron analizadas: (1) variables maternas y del parto: edad (años), número de embarazos, presentación fetal, tiempo del trabajo de parto, vía de naci- miento (vaginal o cesárea), presencia de meconio, realización de episiotomía (medio-lateral derecha e izquierda mediana); (2) del recién nacido: peso al nacer (gramos); sexo; talla $(\mathrm{cm})$; perímetro cefálico $(\mathrm{cm})$; perímetro torácico $(\mathrm{cm})$; raza; Apgar 1ำ y 5 minuto; gasometría fetal ( $\mathrm{pH}$ y exceso de base) obtenida de la arteria umbilical, luego después de la expulsión; adecuación del peso fetal (adecuado, pequeño y grande para la edad gestacional); (3) clasificación del traumatismo al nacer, injurias fetales de acuerdo con la gravedad: trauma tipo I o leve (hemorragia conjuntival, lesiones de la piel, lesiones de tejidos blandos, fractura de clavícula); trauma tipo II o moderados (parálisis braquial y facial, cefalohematoma); trauma tipo III o graves (anoxia); (4) sitio del trauma (cabeza, rostro, brazo); (5) tratamiento de la lesión fetal (inmovilización, sutura simples, acompañamiento) $(1,2)$. Los factores de riesgo pesquisados fueron: macrosomia, vía de parto, multiparidad, presentación fetal, edad materna, expulsivos prolongados.

Los análisis estadísticos fueron realizados con el programa SPSS versión 19.0. Fueron efectuados análisis bi-variados entre los grupos con los tests chi-cuadrado para comparación de variables categóricas y de Mann-Whitney y t de Student para variables continuas, dependiendo de la distribución. Los factores que obtuvieron nivel significativo $<0,10$ en los análisis bivariado fueron insertas en la regresión logística. Se utilizó el modelo de entrada por bloques (block entry) para selección de la regresión. Fueron considerados como control los 115 nacimientos subsecuentes a los casos estudiados. El análisis de riesgo se efectuó mediante el cálculo de Odds Ratio (OR) con intervalo de confianza del 95\% (IC 95\%).

\section{RESULTADOS}

En el período citado nacieron 2.137 niños, 26 $(1,2 \%)$ sufrieron trauma al nacer. Entre ellos, la fractura de clavícula fue la lesión más frecuentemente observada $(n=14 ; 53,8 \%)$, seguida del cefalohematoma ( $n=5 ; 19,2 \%)$. Los demás tipos de lesiones que colaboraron para el total de la muestra, cada una de ellas con un caso, fueron: lesiones de tejidos blandos, lesión de la piel, bolsa serosangüínea, hemorragia conjuntival, parálisis braquial bilateral, parálisis facial y anoxia intraparto. Solamente un RN, que peso $3.815 \mathrm{~g}$ y que tuvo calificación de Apgar 0 y 1 , en el $1^{\circ}$ y $5^{\circ}$ minutos, respectivamente, presentó anoxia y lesión de tipo III o grave. El nacimiento de ese concepto estuvo relacionado con la rotura del útero, durante tentativa de parto vaginal seguido de una cesárea previa. Con relación 
a los tratamientos propuestos, la inmovilización fue la conducta escogida ( $n=14 ; 53,8 \%)$, seguida de acompañamiento clínico $(n=11 ; 42,3 \%)$ y de sutura simples $(n=1 ; 3,8 \%)$. La Tabla I presenta la distribución de esas variables.

La Tabla II presenta el análisis bivariado de las variables maternas. Se puede observar significancia estadística en lo que se refiere a la vía de parto. En la Tabla III, son presentadas las variables fetales; se observa diferencia significativa en lo referen-

\section{Tabla I}

DISTRIBUCIÓN DE LOS TIPOS DE TRAUMATISMO AL NACIMIENTO Y DEL TRATAMIENTO PROPUESTO

\begin{tabular}{lcc}
\hline Variables & $\mathrm{n}$ & $\%$ \\
\hline Muy leve $(\mathrm{n}=2)$ & & \\
Hemorragia conjuntival & 1 & 4 \\
Tipo I o leve ( $\mathrm{n}=16)$ & & \\
Lesión de la piel & 1 & 4 \\
Lesión de tejido blando & 1 & 4 \\
Bolsa serosanguínea & 1 & 4 \\
Fractura de clavícula & 14 & 53,8 \\
Tipo II o moderado ( $\mathrm{n}=7)$ & & \\
Parálisis braquial bilateral & 1 & 4 \\
Parálisis facial & 1 & 4 \\
Cefalohematomas & 5 & 19,2 \\
Tipo III o grave ( $\mathrm{n}=1)$ & & \\
Anoxia & 1 & 4 \\
Sitio del trauma & & \\
Cabeza & 9 & 34,6 \\
Rostro & 1 & 4 \\
Brazo & 16 & 61,4 \\
Tratamiento de la lesión & & \\
Sutura simples & 1 & 4 \\
Inmovilización del área afectada & 14 & 53,8 \\
Acompañamiento & 11 & 42,2 \\
\hline
\end{tabular}

te al peso y perímetro torácico, ambos relacionados a los fetos de mayor peso. No hubo diferencia significativa en las variables gasométricas de los RN (Tabla IV).

En la análisis de regresión logística, las únicas variables independientes asociadas al traumatismo al nacimiento fueron parto vaginal (OR-A: 11,08; IC95\%: 2,45-49,98; $p=0,002)$ y perímetro torácico $\geq 33$ cm (OR-A: 3,36; IC95\%: 1,35-9,73; $p=0,010$ ) (Tabla V).

\section{DISCUSIÓN}

Aunque hay poca información en la literatura, las conductas estandarizadas y protocolos bien definidos, ejecutados por profesionales capacitados, tienden a reducir de forma significativa la incidencia del traumatismo al nacimiento. Sin embargo, merece énfasis que estos eventos pueden ocurrir igual en manos expertas y en maternidades de referencia (3-9).

La incidencia de los diferentes tipos de traumatismo al nacimiento presentan una amplia variación dentro de la literatura recopilada. Baskett y cols (3), presentan incidencia de $2 \%$, mientras que Mosavat y cols (6), citan una tasa de $0,8 \%$ del total de nacimientos a término, siendo el tipo más común el cefalohematoma $(37,3 \%)$, seguido de la fractura de clavícula (11,2\%). En este último estudio, fue identificado que el nacimiento por cesárea constituye un factor protector para el traumatismo al nacimiento $(1,3 \%$ vs. $0,1 \% p=0,0001)$, semejante a los datos obtenidos en nuestro estudio. Para Barrientos y cols (15), la frecuencia de trauma al nacimiento fue de $1,44 \%(n=309)$, después del seguimiento de cinco años. En ese estudio, los autores citan como principal trauma la fractura de clavícula $(n=107$ $34,6 \%)$, seguido de cefalohematoma $(n=105 ; 34 \%)$.

Tabla II

ANÁLISIS DE LAS VARIABLES MATERNAS ASOCIADAS AL TRAUMATISMO AL NACER

\begin{tabular}{lccc}
\hline Variables maternas & $\begin{array}{c}\text { Caso (\%) } \\
n=26\end{array}$ & $\begin{array}{c}\text { Controles (\%) } \\
n=115\end{array}$ & Valor $p$ \\
\hline \hline Edad (media en años) * & $25,1 \pm 5,7$ & $25,0 \pm 6,8$ & 0,953 \\
Nuliparidad ** $_{\text {Presentación cefálica ** }}^{* *}$ & 44,6 & 96,5 & 0,271 \\
Período expulsivo (min) ** & 92,3 & $5,0(3,8-11,3)$ & 0,895 \\
Parto vaginal ** & $10,0(10,0-20,0)$ & 53,0 & 0,011 \\
Uso de fórceps ** & 92,3 & 6,1 & $<0,0001$ \\
Líquido amniótico meconial ** & 3,8 & 18,3 & 0,656 \\
Episiotomía ** & 19,2 & 44,3 & 0,908 \\
\hline
\end{tabular}

* Test t de Student; ${ }^{\star \star}$ Test chi-cuadrado; ${ }^{\star \star \star}$ Test de Mann-Whitney. 


\section{ANÁLISIS DE LAS VARIABLES FETALES ASOCIADAS AL TRAUMATISMO AL NACER}

\begin{tabular}{|c|c|c|c|}
\hline Variables fetales & $\begin{array}{c}\text { Caso (\%) } \\
n=26\end{array}$ & $\begin{array}{c}\text { Controles (\%) } \\
n=115\end{array}$ & Valor $\mathrm{p}$ \\
\hline Peso $(g)$ * & $\begin{array}{c}3.440,0 \\
(3.061,3-3.875,0)\end{array}$ & $\begin{array}{c}3.045,0 \\
(2.555,0-3.430,0)\end{array}$ & 0,005 \\
\hline Género masculino ** & $53,8 \%$ & $50,4 \%$ & 0,754 \\
\hline Talla $(\mathrm{cm}) *$ & $49,8(47,6-51,0)$ & $48,0(46,0-50,0)$ & 0,022 \\
\hline Perímetro cefálico $(\mathrm{cm})$ * & $34,0(33,0-35,0)$ & $32,4(32,0-34,5)$ & 0,020 \\
\hline Perímetro torácico $(\mathrm{cm})^{*}$ & $34,0(32,4-35,0)$ & $32,0(30,0-33,5)$ & 0,001 \\
\hline Adecuación del peso fetal ** & & & 0,223 \\
\hline PEG & 7,7 & 7,2 & \\
\hline AEG & 61,5 & 76,6 & \\
\hline GEG & 30,8 & 16,2 & \\
\hline Apgar 1er minuto * & $7(4-9)$ & $8(7-9)$ & 0,022 \\
\hline Apgar $5^{\circ}$ minuto * & $9(8-10)$ & $9(9-9)$ & 0,459 \\
\hline
\end{tabular}

* Test de Mann-Whitney; ${ }^{* \star}$ Test chi cuadrado; PEG: Pequeño para edad la gestacional; AEG: Adecuado para la edad gestacional; GEG: Grande para la edad gestacional.

\section{Tabla IV \\ ANÁLISIS COMPARATIVO DE LAS VARIABLES GASOMÉTRICA ENTRE LOS GRUPOS}

\begin{tabular}{lccc}
\hline Variables & $\begin{array}{c}\text { Casos } \\
\text { (media } \pm \mathrm{DE})\end{array}$ & $\begin{array}{c}\text { Controles } \\
(\text { media } \pm \mathrm{DE})\end{array}$ & $\mathrm{P}$ \\
\hline $\mathrm{pH}$ & $7,26 \pm 0,08$ & $7,21 \pm 0,1$ & 0,058 \\
$\mathrm{pCO} 2$ & $43,2 \pm 13,2$ & $51,9 \pm 13,8$ & 0,023 \\
$\mathrm{pO} 2$ & $17,9 \pm 5,7$ & $19,7 \pm 9,8$ & 0,495 \\
$\mathrm{HCO} 3$ & $20,4 \pm 4,6$ & $20,1 \pm 3,5$ & 0,808 \\
Exceso de base & $-6,3 \pm 4,8$ & $-7,9 \pm 4,1$ & 0,198 \\
Saturación de O2 & $21,3 \pm 11,6$ & $24,0 \pm 18,3$ & 0,580
\end{tabular}

Test t de Student. DE: desvío estándar.

Tabla V

REGRESIÓN LOGÍSTICA CON FACTORES INDEPENDIENTES ASOCIADOS AL TRAUMATISMO AL NACER

\begin{tabular}{lcccc}
\hline Variables & B & OR-A & IC95\% & Valor $\mathrm{p}$ \\
\hline Parto vaginal & 2,405 & 11,08 & $(2,5-49,9)$ & 0,002 \\
Perímetro torácico & 1,289 & 3,62 & $(1,4-9,7)$ & 0,010 \\
$\geq 33 \mathrm{~cm}$ & & & & \\
\hline
\end{tabular}

En nuestro estudio fue identificada una incidencia de traumatismo clavicular similar de 1,2\%.

Ha sido citado que la calificación de Apgar igual o menor a siete en el primer minuto de vida puede indicar acidosis fetal intraparto y la naturaleza traumática de los partos (16). Nuestro estudio observó una tendencia en ese sentido, aunque, el pequeño tamaño de la muestra probablemente haya influenciado en el resultado final. La literatura revisada no presenta datos relativos a la gasometría de la arteria umbilical obtenida en el nacimiento y asociación con los traumatismos al nacimiento. El análisis del estudio en cuestión y los datos obtenidos no presentaron una asociación significativa con trauma al nacimiento.

Al compararse el grupo caso y control, no se identificó asociación estadísticamente significativa entre las variables edad materna $(25,1 \pm 5,7$ años vs. $25,0 \pm 6,8$ años; $p=0,953)$ y paridad $[1,1(0-6)$ vs. $1,0(0-7)]$, como factores de riesgo. No hay consenso en la literatura revisada la relación trauma obstétrico relacionado a la variable edad materna (14). Se destaca que las mujeres con edad superior a 35 años se relacionan a mayor número de casos de parálisis del plexo braquial, aunque no haya sido observada diferencia estadísticamente significativa entre las primíparas y las multíparas (17).

La vía de parto vaginal, analizada por regresión logística, mostró como variable independiente para trauma al nacer (OR-A: 11,08; IC95\%: 2,5-49,9; $\mathrm{p}=0,002)$; por otro lado, la operación cesárea fue considerada factor de protección para injurias fetales leves y graves. Vale resaltar que los traumatismos también pueden ocurrir por ocasión de partos abdominales, más frecuentemente en cirugías de emergencia (11). Para algunos autores, la incidencia de traumatismos relativa a la vía de parto no presentó diferencia estadística, cuando el parto vaginal ocurrió con el auxilio de fórceps o vacuo ex- 
tractor $(2,8 \%$ vs. $1,3 \%, p=0,303)(6,7,12,17)$.

Probablemente debido al tamaño de nuestra muestra, la duración del período expulsivo ( $2^{\circ}$ período del trabajo de parto), no se mostró estadísticamente diferente comparado con el grupo control $[10,0(10,0-20,0 \mathrm{~min})$ y $5,0(3,75-11,25 \mathrm{~min})$, respectivamente, $(p=0,011)]$. Es citado en la literatura que la mitad de las lesiones del plexo braquial fetal ocurren después de partos vaginales relacionados con el expulsivo mayor a 30 minutos $(11,17)$.

Publicaciones sobre este tema muestran que el peso fetal es el mayor factor asociado al trauma al nacer y a lesiones maternas. En nuestro estudio fue analizado, también, la estatura fetal, que no presentó significancia estadística, aunque las mayores estaturas se asocien a mayores pesos fetales. Fetos macrosomicos están frecuentemente involucrados con lesiones de neonatos y maternas $(18,19)$. En nuestra casuística, el peso medio de los neonatos del grupo caso fue de $3.440 \mathrm{~g}(3.061,5$ $\mathrm{g}-3.875 \mathrm{~g})$, y en el grupo control de $3.045 \mathrm{~g}$ (2.555 $\mathrm{g}-3.430 \mathrm{~g}$ ), diferencia estadísticamente significativa $(p=0,005)$. Esa variable no presentó asociación significativa con la fractura de clavícula. Se estima que ese resultado se debe al pequeño tamaño de la muestra. Mientras que el perímetro torácico aumentado $34 \mathrm{~cm}(32,4-35 \mathrm{~cm})$ vs. 32 (30 - 33,5 $\mathrm{cm})$, característico de fetos mayores, fue considerado como factor independiente asociado a la lesión fetal. Baskett y cols (3), cuyo estudio incluye 2.385 casos, citan que el principal trauma al nacer fue la fractura de clavícula por peso fetal aumentado y la distocia de hombro.

\section{CONCLUSIÓN}

La incidencia de traumatismos al nacimiento fue de $1,2 \%$. Los factores de riesgo que se asociaron significativamente a trauma fetal fueron el parto vaginal y el perímetro torácico igual o superior a $33 \mathrm{~cm}$.

\section{REFERENCIAS}

1. Berhman RE, Kliegman RM. Nelson - Essentials of Pediatrics. New York: WB Saunders, 2002.

2. Costa HPF. Traumatismos de parto. In: Perinatologia. Fundamentos e Prática. 1ํㅡㄹ ed. São Paulo: Sarvier, 2002. p. 476-85.
3. Baskett TF, Allen VM, O'Connell CM, Allen AC. Fetal trauma in term pregnancy. Am J Obstet Gynecol 2007;197:499.e1-7.

4. Zhang X, Decker A, Platt RW, Kramer MS. How big is too big? The perinatal consequences of fetal macrosomia. Am J Obstet Gynecol 2008;198:517.e1-6.e1-7.

5. Madi JM, Rombaldi RL, Oliveira Filho PF, Araújo BF, Zatti $\mathrm{H}$, Madi SRC. Fatores maternos e perinatais relacionados à macrossomia fetal. Rev Bras Ginecol Obstet 2006;28:232-7.

6. Mosavat SA, Zamani M. The incidence of birth trauma among live born term neonates at a referral hospital in Rafsanjan, Iran. J Matern Fetal Neonatal Med 2008;21:337-9.

7. Awari BH, Al-Habdan I, Sadat-Ali M, Al-Mulhim A. Birth associated trauma. Saudi Med J 2003;24:672-4.

8. Doumouchtsis SK, Arulkumaran S. Head injuries after instrumental births. Clin Perinatol 2008;35:69-83.

9. Clark SL, Vines VL, Belfor MA. Fetal injury associated whit routine vaccum use during cesarean delivery. Am J Obstet Gynecol 2008;198:e4.

10. Babović I, Plesiinac S, Radojicić Z, Opalić J, Argirović R, Mladenović-Bogdanović Z, Plećas D. Vaginal delivery versus cesarean section for term breech delivery. Vojnosanit Pregl 2010;67:807-11.

11. Backe B, Magnussen EB, Johansen OJ, Sellaeg G, Russwurm H. Obstetric brachial plexus palsy: a birth injury not explained by the known risk factors. Acta Obstet Gynecol Scand 2008;87:1027-32.

12. Gurewitsch ED, Allen RH. Shoulder dystocia. Clin Perinatol 2007;34:365-85.

13. Parker LA. Part 1: early recognition and treatment of birth trauma: injuries to the head and face. Adv Neonatal Care 2005;5:288-97.

14. García H, Rubio-Espíritu J, Islas-Rodríguez MT. Risk factors for birth injuries. Rev Invest Clin 2006;58:416-23.

15. Barrientos G, Cervera $P$, Navascués J, Sánchez R, Romero R, Pérez-Sheriff V, et al. Obstetric trauma. A current problem? Cir Pediatr 2000;13:150-2.

16. Baud $O$. Neonatal outcomes after instrumental vaginal delivery. J Gynecol Obstet Biol Reprod 2008;37 Suppl 8:S260-8.

17. Hudić I, Fatusić Z, Sinanović O, Skokić F. Etiological risk factors for brachial plexus palsy. J Matern Fetal Neonatal Med 2006;19:655-61.

18. Dessole S, Cosmi E, Balata A, Uras L, Caserta D, Capobianco $\mathrm{G}$, et al. Accidental fetal lacerations during delivery: experience in an Italian Level III university hospital. Am J Obst Gynecol 2004;191:1673-7.

19. Akin $Y$, Cömert S, Turan C, Piçak A, Ağzikuru T, Telatar B. Macrosomic newborns: a 3-year review. Turk J Pediatr 2010;52:378-83. 